\title{
SUBSET SELECTION FOR LANDMARK MODERN AND HISTORIC IMAGES
}

\author{
Heider K. Ali ${ }^{1}$ and Anthony Whitehead ${ }^{2}$ \\ ${ }^{1}$ Carleton University, Systems \& Computer Engineering Department , \\ Ottawa, ON, K1S 5B8, Canada \\ heiderasce.carleton.ca \\ ${ }^{2}$ Carleton University, School of Information Technology, \\ Ottawa , ON, K1S 5B8, Canada \\ Anthony. Whiteheadecarleton.ca
}

\begin{abstract}
An automatic mechanism for the selection of image subset of modern and historic images out of a landmark large image set collected from the internet is designed in this paper. This selection depends on the extraction of dominant features using Gabor filtering. These features are selected carefully from a preliminary image set and fed into a neural network as a training set. The mechanism collects a raw large set of landmark images containing modern and historic images and non-landmark images as well, process these images, and finally classify them as landmark and non-landmark images. The classification performance highly depends on the number of candidate features of the landmark.
\end{abstract}

\section{KEYWORDS}

Feature Extraction, Neural Networks, Gabor Filters, Subset Selection, Image Categorization

\section{INTRODUCTION}

Classification of a large set of images containing both modern and historic images which leads to a selection of a subset of these images which fulfills certain technical standards is based completely on the detection and extraction of image features. Feature extraction has been received significant effort of researchers because of its great role in computer vision, image processing, and robotics fields [1]. Many standard feature extraction techniques have been applied to images to achieve optimal extraction performance and to maintain robustness by addressing the varying spatial resolutions, illumination, observer viewpoint, and rotation $[2,3,4$, 5]. Some success has been found by using a hybrid of standard techniques [6]. These techniques have achieved partial success in the feature matching in a mixed set of modern and historic images.

Object recognition techniques based on Gabor filters for feature extraction shown moderate success in extracting fundamental frequencies which represents the shape of an object [7]. Because the Gabor filters act as edge, shape and line detectors, as well as the tuning flexibility with different orientations and frequencies [8], these filters are often applied in a wide range of applications such as texture segmentation and classification [9], face recognition [10], fingerprint matching [11], and motion tracking [12].

In our case of mixed modern and historic images captured with cameras of different technologies under different environmental conditions, it is necessary to select the most appropriate image

Natarajan Meghanathan et al. (Eds) : COSIT, SEC, SIGL, AIAPP - 2015

pp. 69-79, 2015. (C) CS \& IT-CSCP 2015

DOI : $10.5121 /$ csit.2015.50607 
subset from a huge image set with significant redundancy. This task can be successfully achieved by using Gabor filters for feature extraction which offer promising prospects in object recognition where the scale, rotation, translation and illumination invariant recognition can be realized within a reasonable computational time limit [8]. The selected features are trained on a neural network to recognize and extract the true features and to select the images of highest similarity.

This paper presents an automatic matching mechanism to select an image subset of landmarks out from a huge image set downloaded or collected from image websites like Google images or Flickr.

\section{RELATED WORK}

Selection of image subset from a large set depends widely on the image type and contents. This image nature reflected widely on the criteria of subset selection, and hence, on the efforts of researchers in image categorization and classification. Thorough analysis of image contents has to be done to extract the salient features and characteristics that characterize an image set. This extraction relies on the conditions of the image were taken in [1] .Traditional image set selection and classification is mainly relying on the analysis of the low-level features of the image to get high-level content semantics [14]. To optimize the feature detection and extraction process, the researchers worked to support their efforts by non-traditional techniques. Machine learning techniques like artificial neural networks (ANN) has obtained popularity in image subset selection [15].

Xiong et. Al. [16] proposed a BP neural network to improve the performance of image selection by segmenting and clustering the image into several visual objects and building the total feature vector, while $\mathrm{Xu}$ and $\mathrm{Qu}$ [17] developed a method based on feature matching similarity and frequency-inverse document frequency (TF-IDF) value of region is proposed. Multi-scale features used by $\mathrm{Li}$ and Zhao[18] in medical image classification and the classifiers use a set of complementary image features in various scales to compare the results of classification process. To optimize the feature selection process for a robust image classification, Al-Sahaf et. al. [19] introduced a Two-Tier Genetic Programming (GP) based image classification method which works on raw pixels rather than high-level features.

\section{GABOR FILTERS}

Gabor filters are based on the Gabor wavelets which are formed from two components, a complex sinusoidal carrier placed under a Gaussian envelope. These wavelets are based on the Gabor elementary function presented by D. Gabor in 1946 [13]. Many forms of the 2-D Gabor filter have been given. The 2-D Gabor filter $G(x, y)$ can be defined as [7]:

$$
G\left(x, y=e^{-\left(\alpha^{2} x_{p}^{2}+\beta^{2} y_{p}^{2}\right)} e^{j 2 \pi f_{0} x_{p}}\right.
$$

Where $\alpha$ is the time duration of the Gaussian envelope and the plane wave, $\mathrm{f}_{0}$ is the frequency of the carrier, $x_{p}=x \cos \theta+y \sin \theta, y_{p}=-x \sin \theta+y \cos \theta$ and $\Theta$ and $\beta$ are the sharpness values of the major and minor axes of the Gaussian envelope. Gabor filters can be used effectively to make the classification with varying capture conditions [8]. In this work we examine the effectiveness of Gabor filters in a situation where other techniques have failed significantly [6]. Specifically, by using an image set of a landmark containing both historic and modern images with different objects captured by different technologies widely spread throughout time. 


\section{NEURAL NETWORKS}

A multi-layer feed-forward neural network has been used in training a data set on input patterns. A three layer network was used: the input layer, a hidden layer and the output layer. For the input layer, the input feature vectors comes from the Gabor filter feature extraction stage and consists of 100 neurons applied to the neural network. These vectors are processed in the hidden layer using the scaled conjugate gradient method as the training method and the mean square error with regularization as the performance function to adjust the network output to be in the range -1.0 to 1.0. The output layer size depends on the obvious candidate features in each landmark. The following figure shows a typical multi-layer feed-forward neural network.

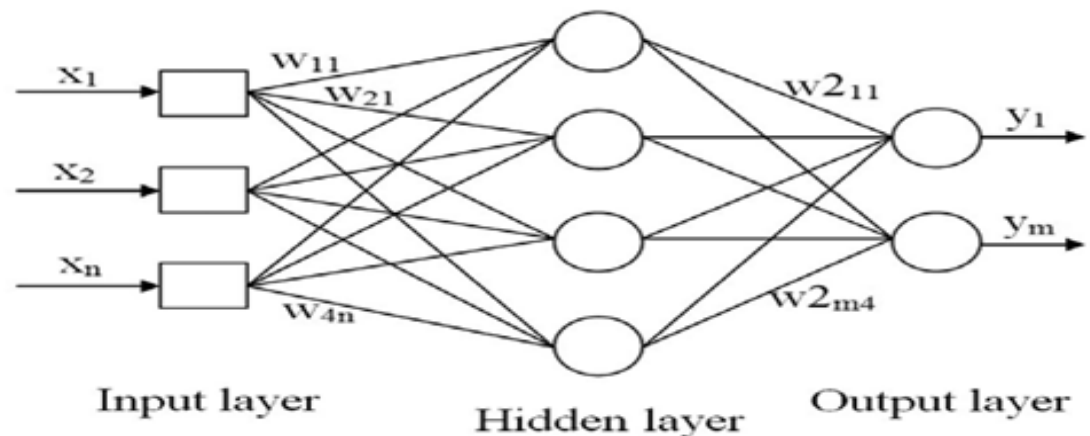

Figure 1. Multi-layer feed-forward neural network

\section{DATA SUBSET SELECTION}

\subsection{Data sets}

The data sets are divided into two categories: the preliminary data sets and the actual dataset. The preliminary data sets are set of modern and historic images for five landmarks. Every data set contains 300-350 images. This data set is primarily used as a training set to the neural network after the Gabor features has been extracted and fed into the neural network. Figure 2 shows some the images of the preliminary data set for Eiffel tower and Coliseum landmarks. Images of other three are shown in Appendix 1.

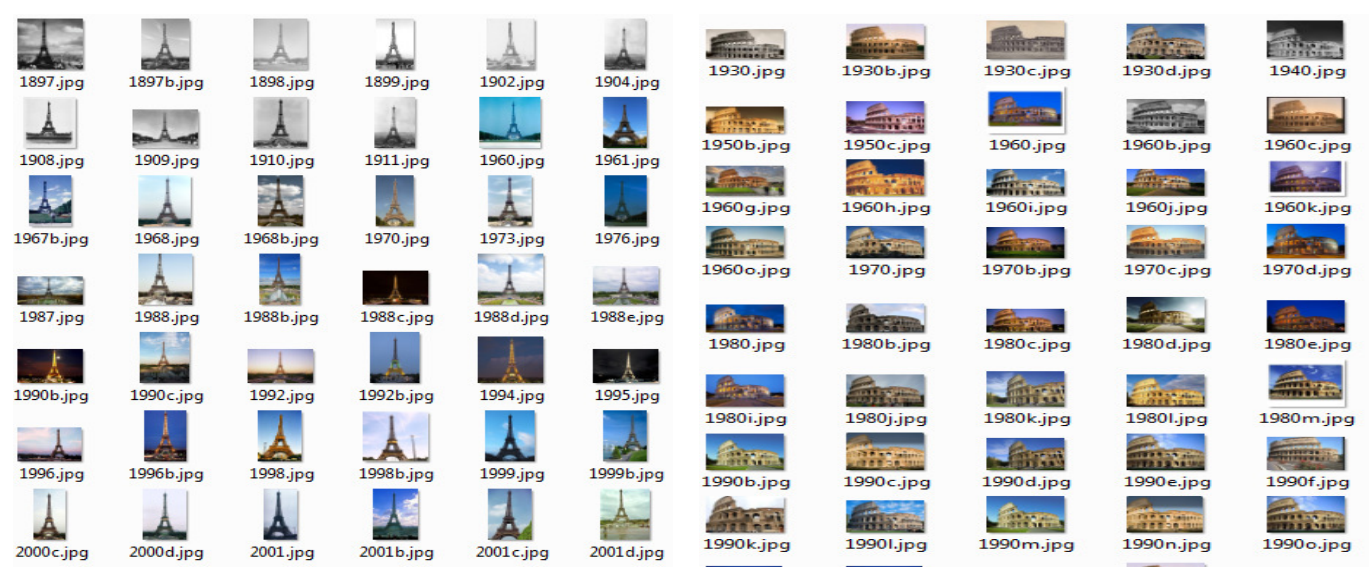

(a)

(b)

Figure 2. Examples of modern and historic images of many landmarks (a) Eiffel tower in Paris (b) Coliseum of Rome 
To prepare these images for the next processing steps, these images have to be converted into gray scale images and resized to a common size. To overcome the problems of variations in brightness and contrast, as well as, the different lighting conditions, these images have to be preprocessed. To compensate for the effects of brightness and contrast, histogram equalization [20] was used. Specifically, adaptive histogram equalization (AHE) is applied to correct the problems of different lighting conditions. AHE computes many histograms; each histogram corresponds to a specific sector of the image and uses them to redistributes the image brightness values [21]. Finally, the input values are normalized to a range of $[-1,1]$.

The actual data set is comprised of sets of modern and historic images of five landmarks. Every set is 1000-3000 images gathered from Google Image Search. These images are placed in a folder, resized, converted to gray scale, and preprocessed to compensate for contrast and lighting problems. These images are applied to the system to classify them as matching or non-matching depending on the Gabor features of every landmark.

\subsection{Feature extraction by Gabor filtering}

To extract the image Gabor filters, it is required to create the Gabor wavelets. These wavelets are created using equation (1) above by using three control factors to create the Gabor kernels. Figure 3 shows the Gabor wavelets generated with three control parameters: scale, frequency and orientation.

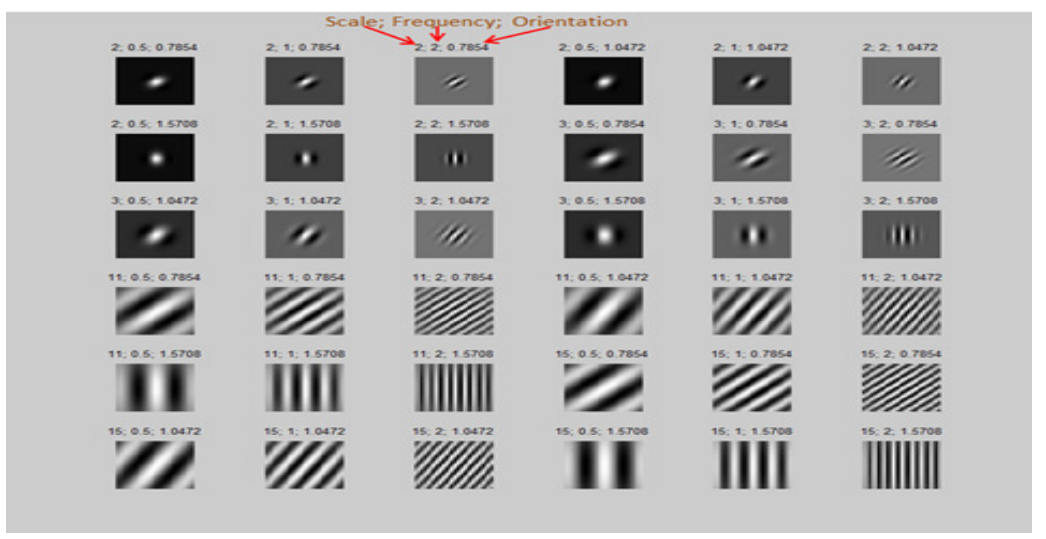

Figure 3. Gabor wavelets created using three control parameters

By applying these kernels on an image, we get the Gabor wavelets family shown in figure 4 .

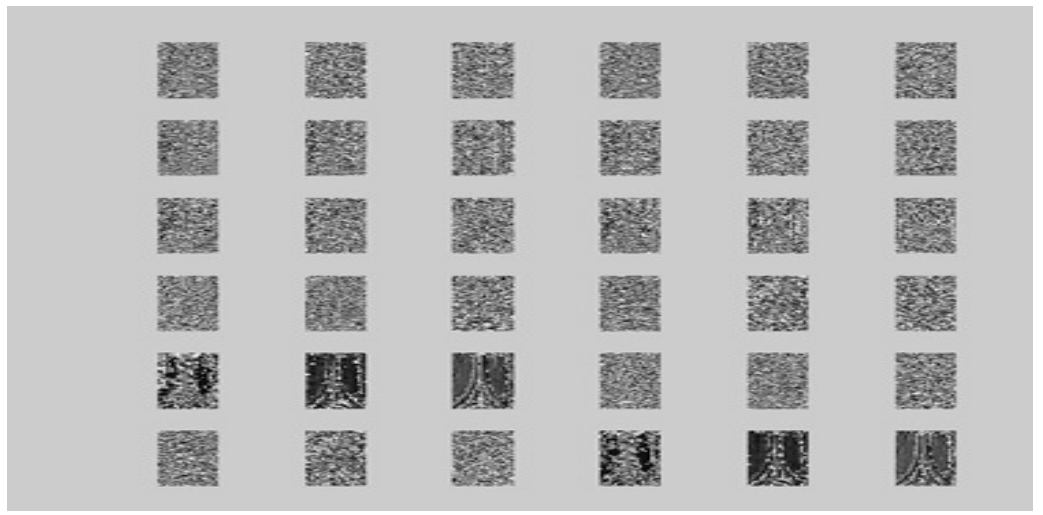

Figure 4. Gabor family 


\subsection{Neural network training}

In the training phase of the neural network two sets of images are applied, a feature image set and non-feature image set. The feature image set consists of 100-150 images containing the feature to be tested, while the non-feature image set is 50 non feature images. Feature images completely depend on the landmark images being investigated. To facilitate the classification process, all landmark images are studied carefully to select the highest detectable features with Gabor filtering to be the candidate features. For example, the Eiffel tower images contain two regions which are assumed to have accurately detectable features. The image sets of these regions are fed into the neural network as the feature image set. For Eiffel tower landmark, the network output neurons will set to two. These outputs appear in a mutually exclusive basis, i.e. if the features exist in an image, one of the outputs will be greater than 0.8 or less than -0.5 and vice versa.

The image classification process described in algorithm 1 is shown in in figure 7 . By a careful study the image set of Eiffel tower landmark; as an example, two features are nominated as candidate features that lead to a reasonable extraction and hence; a good classification. The training image sets of these two areas are fed into the neural network as training sets. Figure 5 shows a sample image of Eiffel tower, the two candidate feature regions, and the training sets fed into the network. The same process is done on the other four landmarks to select the dominant features and to prepare the training image sets of these features for next steps.

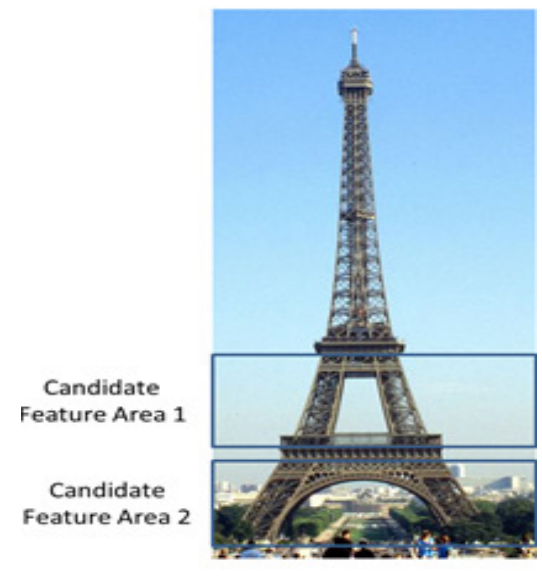

(a)

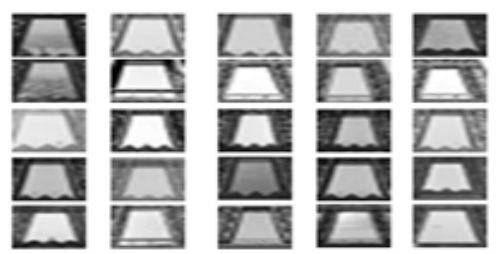

(b)

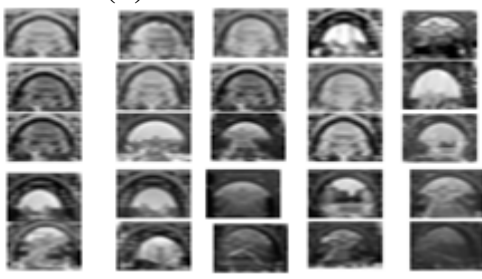

(c)

Figure 5. Eiffel tower image (a) Two candidate feature areas (b) Sample of training set for area 1 (c) Sample of training set for area 2

\subsection{Performing Gabor filtering}

The Gabor filtering is performed by convolving images with Gabor kernels. Generally, convolution can be done using Fast Fourier Transform (FFT). The FFT is done by each multiplying the frequency domain of Gabor kernels with the image pixel by pixel, i.e. dot product. Then, the inverse Fourier Transform (IFFT) has to be done to return the result back into spatial domain. Finally, the feature vector is built by converting the image data which convolved with the Gabor kernels to prepare these images to be trained on the neural network. Figure 6 summarizes the feature extraction process. 


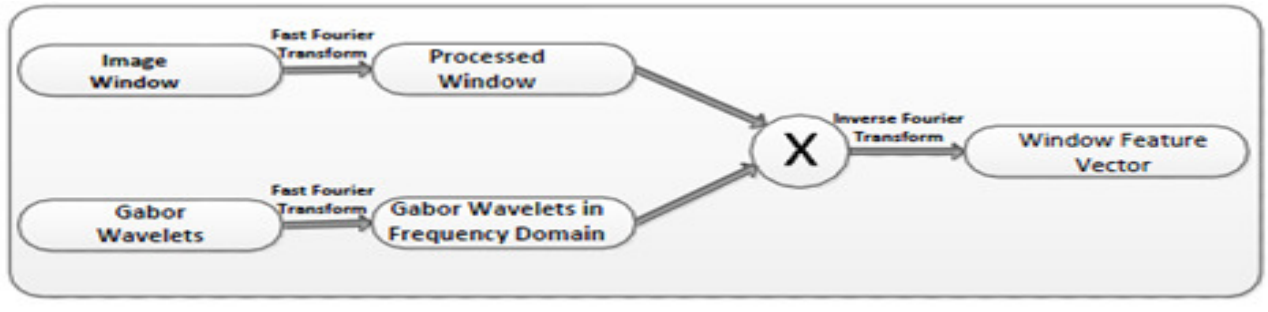

Figure 6. Feature Extraction Process

\subsection{Candidate feature selection}

For every landmark, the candidate feature(s) should be selected prior to training them on the neural network. The selection process depends on the nature of the landmark image. The candidate feature should be unique in the image, i.e. repeated features should not be selected. Also, the features with a larger texture similarity serve better in the selection process.

\subsection{Image matching process}

The image set of a landmark downloaded or collected from the internet is pre-processed by resizing them and adjusting the contrast, brightness and illumination. Then, these images are applied to the neural network recognition phase to classify them as matched images or not. The images are placed in the matched folder or un-matched folder according to algorithm 1 in figure 7.

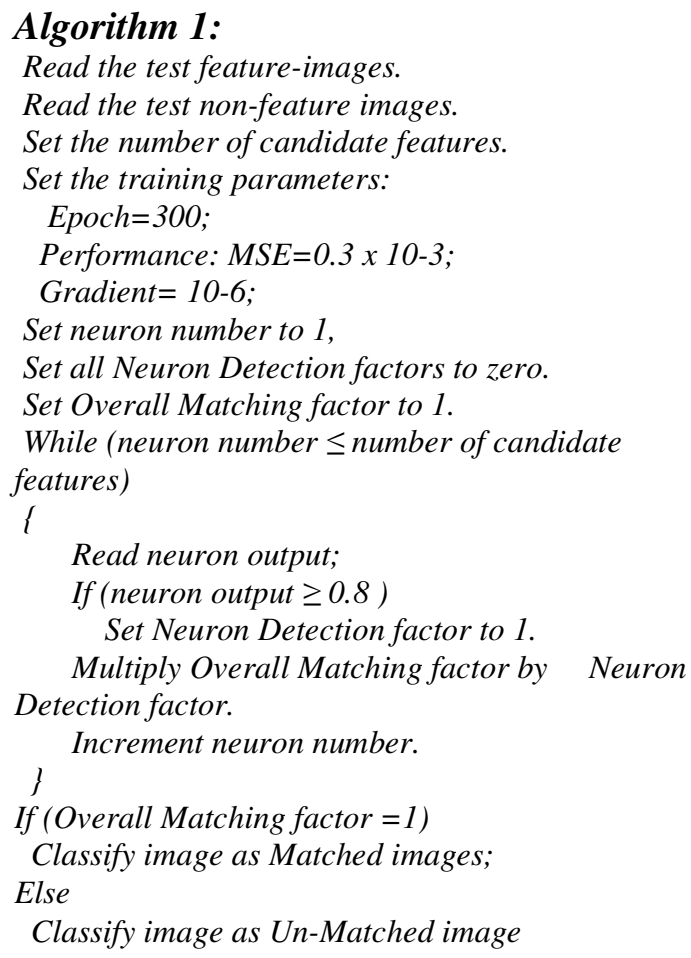

Figure 7. Image classification algorithm 


\subsection{Experimental Results}

The algorithm was applied to two different sets of images of five landmarks, Eiffel Tower, Coliseum of Rome, Pyramids of Giza, Dome Rock, and the Statue of Liberty. The first bunch of image data is the primary data set which comprised of 300-350 images for each of the landmarks. These images are preprocessed to improve their contrast and lighting and then cropped to maintain the most relevant parts.

The features that are most likely detected along with an example of problematic false positives are shown in figure 8 . The false positive problem was partially addressed by selecting the features with highest matching scores. This leads to the selection of most unique features. The landmarks image sets were selected randomly from image websites as a mix of modern and historic images of five landmarks as well as non-landmark images. The landmark images had selected one, two, or three candidate features for evaluation. Each landmark set was applied to the system to classify them into proper folders, namely the landmark image folder and the nonlandmark image folder.
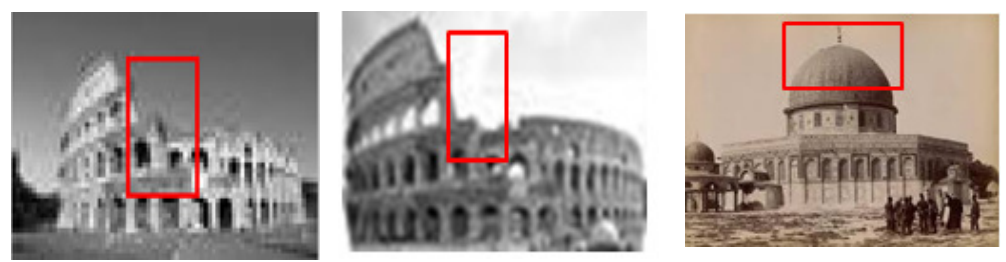

(a)
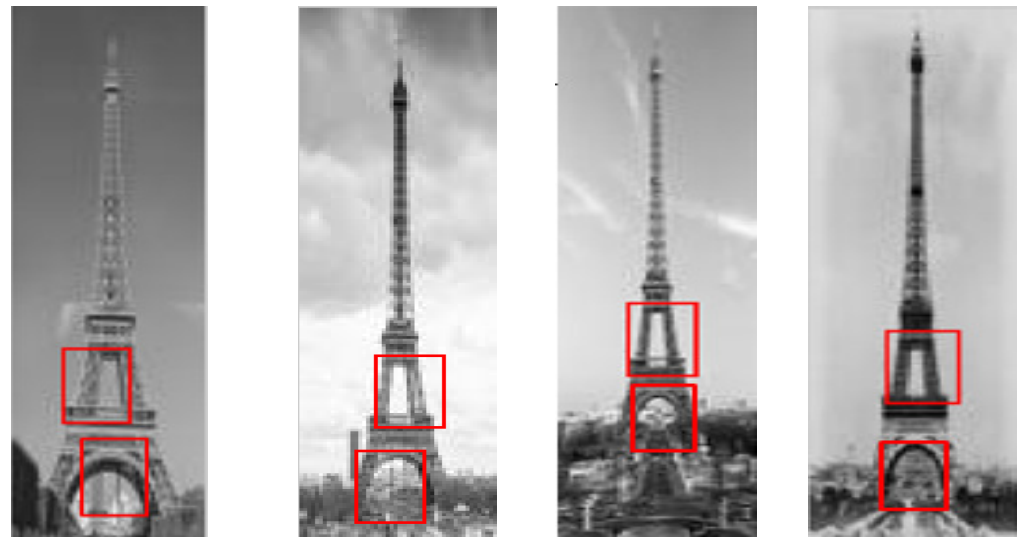

(b)
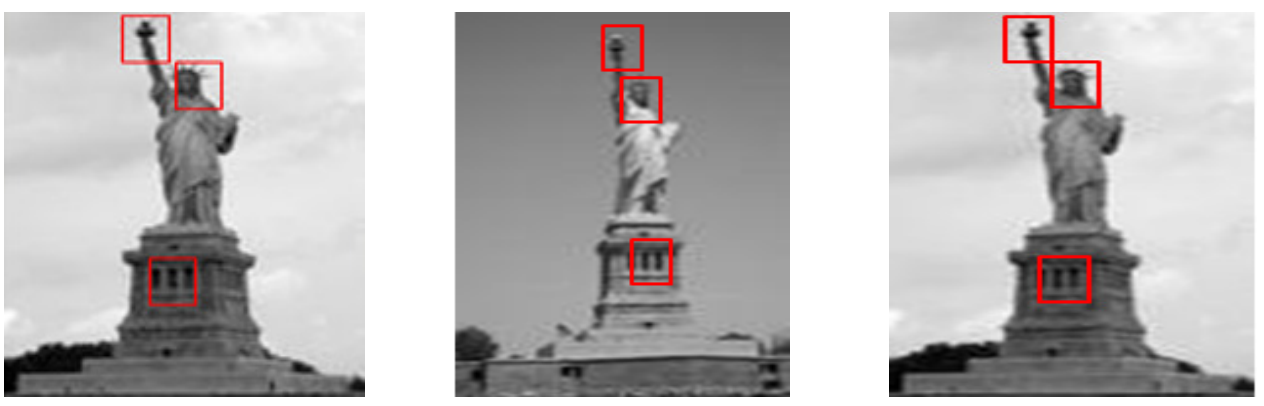

(c)
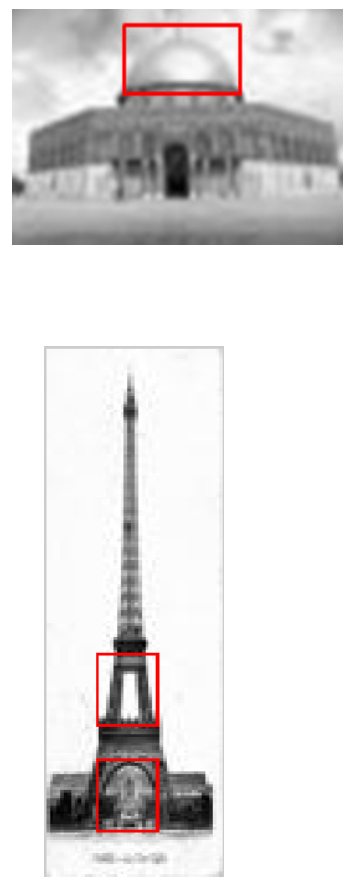

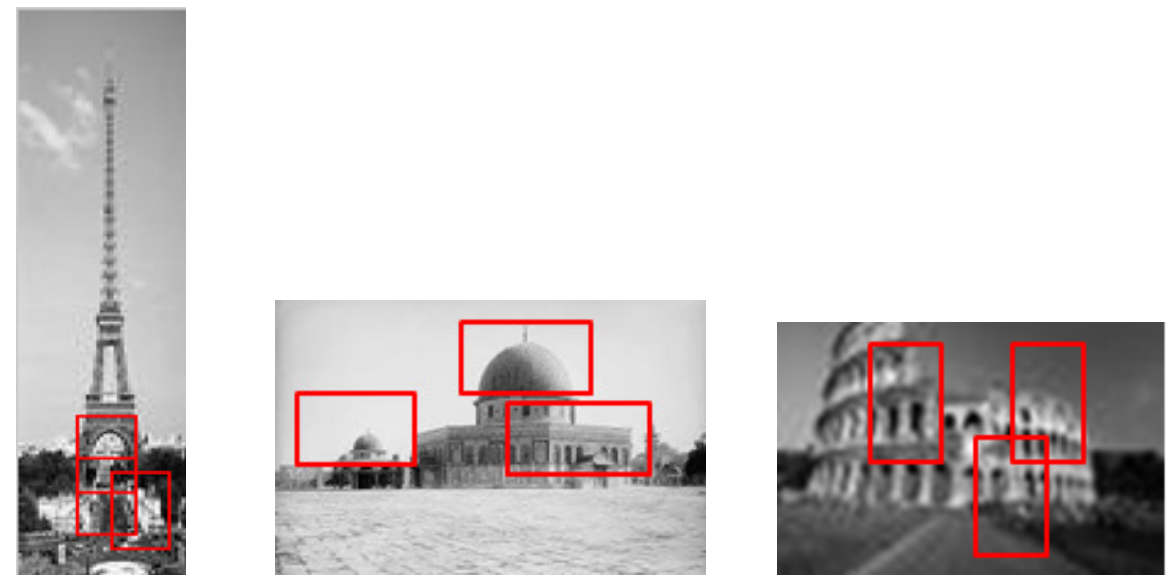

(d)

Figure 8. Feature extraction of images (a) 1-Feature (b) 2-Features (c) 3-Features (d) False positive features

The results are shown in table 1.

Table 1. Applied algorithms results on landmark images collected from the image websites

\begin{tabular}{|c|c|c|c|c|c|}
\hline & $\begin{array}{c}\text { Total } \\
\text { Images }\end{array}$ & $\begin{array}{c}\text { True } \\
\text { Positive }\end{array}$ & $\begin{array}{c}\text { False } \\
\text { Negative }\end{array}$ & $\begin{array}{c}\text { False } \\
\text { Positive }\end{array}$ & $\begin{array}{c}\text { True } \\
\text { Negative }\end{array}$ \\
\hline $\begin{array}{c}\text { One Feature } \\
\text { Image Set }\end{array}$ & 2833 & 1023 & 634 & 819 & 357 \\
\hline $\begin{array}{c}\text { Two Features } \\
\text { Image Set }\end{array}$ & 2960 & 1417 & 780 & 521 & 242 \\
\hline $\begin{array}{c}\text { Three Features } \\
\text { Image Set }\end{array}$ & 2219 & 1569 & 331 & 216 & 103 \\
\hline
\end{tabular}

The results shown in table 1 declare that this algorithm performed effectively on a mixed of modern and historic images of a large number of five landmarks collected randomly from the Google images. Despite the diverse capturing environments of these image in the sense of used camera technology, lighting conditions, and geographical variations of these images, this algorithm exploits the merging of Gabor filtering with the neural network setting to extract the dominant image features and to categorize images as landmark images or no.

To analyze the obtained results of table 1 , the precision and recall metrics were applied to calculate the precision, recall, accuracy and the F-measure factor which is the harmonic mean of precision and recall [22] using the formulas given in table 2.

Table 2. Precision and recall metrics formulas

\begin{tabular}{|c|c|c|c|}
\hline Precision & Recall & Accuracy & F1 Score \\
\hline $\mathrm{tp} /(\mathrm{tp}+\mathrm{fp})$ & $\mathrm{tp} /(\mathrm{tp}+\mathrm{fn})$ & $(\mathrm{tp}+\mathrm{tn}) /(\mathrm{tp}+\mathrm{tn}+\mathrm{fp}+\mathrm{fn})$ & $2^{*} \mathrm{tp} /\left(2^{*} \mathrm{tp}+\mathrm{fp}+\mathrm{fn}\right)$ \\
\hline
\end{tabular}

\subsection{Subset Selection Mechanism Performance and Effectiveness}

The results shown in table 3 reveals that the precision and the accuracy of this mechanism increased as the number of the candidate features of landmark images increased, and the overall performance enhanced by increasing the number of the extracted features. 
The selection mechanism applied on five sets of landmarks images picked up the images with highly detectable features despite the considerable variances in images views, scales, and lighting conditions in an automated manner shows that this subset selection mechanism effectiveness is reasonably accepted and represents a promising image set categorization and selection.

Table 3. Accuracy table of the algorithm

\begin{tabular}{|l|c|c|c|c|}
\hline & Precision & Recall & Accuracy & F1 Score \\
\hline $\begin{array}{l}\text { One } \\
\text { Feature }\end{array}$ & 0.555374593 & 0.617380809 & 0.487116131 & 0.826332795 \\
\hline $\begin{array}{l}\text { Two } \\
\text { Features }\end{array}$ & 0.731166151 & 0.644970414 & 0.560472973 & 1.04267844 \\
\hline $\begin{array}{l}\text { Three } \\
\text { Features }\end{array}$ & 0.878991597 & 0.825789474 & 0.753492564 & 1.482986767 \\
\hline
\end{tabular}

\section{CONCLUSIONS}

Selection of the suitable images from a larger set of a landmark images containing both modern and historic images and captured under different lighting conditions and from different viewpoints represents a very important task when looking to match historical imaginary to modern imaginary. We show that Gabor filtering for such a task is a promising methodology when combined with a Neural Network for categorization. Dominant features from the images of many landmarks were selected as training image set to be fed to the neural network. In the categorization stage, the raw images were applied as input to the neural network. The categorization results have shown the proposed method to be a promising first step in creating historical to modern timeline image sets.

\section{REFERENCES}

[1] M. S. Nixon \& A. S. Aguado,(2012) Feature Extraction \& Image Processing for Computer Vision, $3^{\text {rd }}$ Ed. Academic Press.

[2] M. D. Lowe,( 2004) "Distinctive image features from scale-invariant keypoints", International Journal of Computer Vision, Vol. 60, No. 2, pp. 91-110.

[3] H. Bay, et al.,(2006) "Surf: Speeded up robust features," In European Conference on Computer Vision.

[4] E. Rublee, et al.,(2011) "ORB: an efficient alternative to SIFT or SURF”, In IEEE International Conference on Computer Vision, pp. 2564-2571.

[5] M. Calonder, et al.,(2010) "BRIEF: Binary Robust Inde-pendent Elementary Features", Proceedings of the 11th European Conference on Computer vision: Part IV, pp. 778-792.

[6] H. K. Ali, \& A. Whitehead,(2014)"Feature Matching for Aligning Historical and Modern Images", International Journal of Computer Applications IJCA, Vol. 21, No. 3, pp. 188-201.

[7] J. Kamarainen, et al.,(2002) "Fundamental frequency Gabor filters for object recognition", In Proceedings of the 16th International Conference on Pattern Recognition, pp. 628-631.

[8] S. Berisha,(2009) "Image classification using Gabor filters and machine learning", MSc Thesis, Wake Forest University, Winston-Salem, North Carolina.

[9] A.C. Bovic, et al.,(1990) "Multichannel texture analysis using localized spatial filters", IEEE Trans. Patter Analysis and Machine Intelligence.

[10] Y. B. Jemaa \& S. Khanfir,(2009) "Automatic local Gabor features extraction for face recognition", Inter Journal of Comp. Science and Information Security, Vo. 3, No. 1.

[11] M. U. Munir \& M.. Y. Javed,(2004) "Fingerprint Matching using Gabor Filters", In Proceedings of National Conference on Emerging Technologies.

[12] B.S. Manjunath, et al.,(1996) "A new approach to image feature detection with applications ", Pattern Recognition.

[13] D. Gabor,(1946) “ Theory of communications”, Journal of Inter-national Electrical Engineers, Vo. 93,pp. 427-457. 
[14] S. Chang et. al. (1998)," Semantic Visual Templates: Linking Visual Features to Semantics" In Proceedings of International Conference on Image Processing. pp. 531-535.

[15 ] N. A. Mahmon \& N. Ya'acob,(2014) "A Review on Classification of Satellite Image Using Artificial Neural Network (ANN)", In IEEE 5th Control and System Graduate Research Colloquium. pp. 153157.

[16] Z. Xiong et. al., (2012) " An Algorithm of I mage Classification Based on BP Neural Network", In IEEE International Conference of Computer Science and Automation Engineering (CSAE) . pp. 523526.

[17] D. Xu \& Z. Qu, (2013) "An Image Classification Method Based on Matching Similarity and TF-IDF Value of Region", In Sixth International Symposium on Computational Intelligence and Design. pp. 112-115.

[18] B. Li et. al. (2013) " Multi-Scale Feature Based Medical Image Classification", In $3^{\text {rd }}$ International Conference on Computer Science and Network Technology. pp. 1182-1186.

[19] H. Al-Sahaf et. al., ( 2012) " Extracting Image Features for Classification By Two-Tier Genetic Programming", In IEEE World Congress on Computational Intelligence.

[20] R. C. Gonzalez a\& R. E. Woods,(2008) "Digital Image Pro-cessing", Prentice-Hall, $3^{\text {rd }}$ Edition.

[21] D. J. Ketcham, et al.,(1974) "Image enhancement techniques for cockpit displays", Tech. rep., Hughes Aircraft.

[22] D. M. W. Powers,(2007)"Evaluation: From Precision, Recall and F-Factor to ROC, Informedness, Markedness \& Correlation", Journal of Machine Learning Technologies, Vo. 2, No. 1, pp. 37-63.

\section{APPENDIX 1}

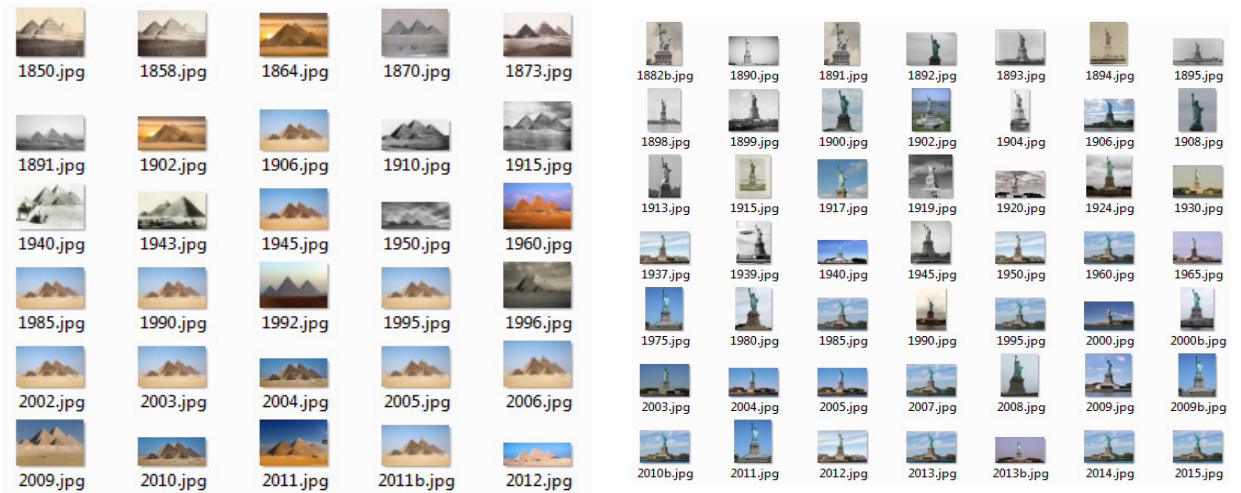

(a)

(b)

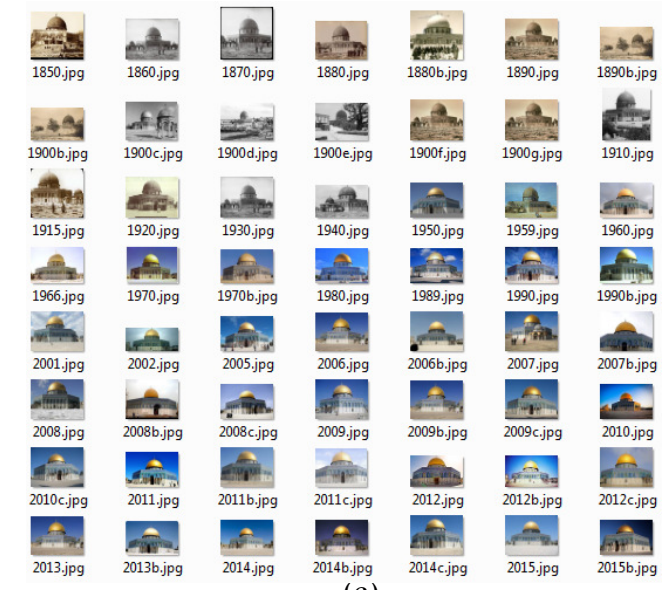

(c)

Figure A-1. Examples of modern and historic images of many landmarks (a) Pyramids of Giza(b) Statue of Liberty (c) Dome of Rock 
AUTHORS

Heider K Ali is doing his $\mathrm{PhD}$ in Electrical and Computer Engineering at Carleton University. He works on the feature matching of modern to historic photos of many worldwide landmarks and the investigation of the problems of dealing with historic photos. He got his MSc in computer engineering in 1991 and BSc in control and systems engineering in 1986 both from the University of Technology, Iraq. His fields of interest are computer vision, panorama building, historic to modern photos matching, software engineering and machine learning.

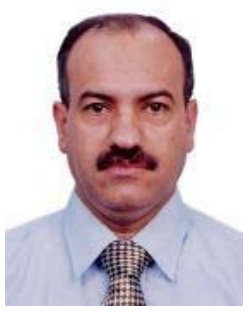

Anthony Whitehead is an Associate Professor and Director of the School of Information Technology at Carleton University. He is also the current Chair of the Human Computer Interaction Program at Carleton University. His research interests can be described as practical applications of machine learning, pattern matching and computer vision as they relate to interactive applications (including games), computer graphics, vision systems and information management.

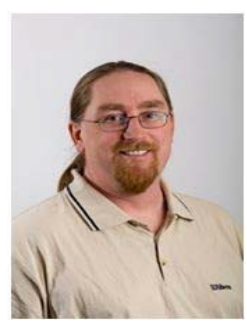

